Stefan Immerfall

\title{
Optionsrechte, Zugehörigkeiten und die Schwierigkeiten supranationaler Systembildung
}

\section{Fragestellungen der historisch-vergleichenden Makrosoziologie Stein Rokkans"}

Zusammenfassung: Stein Rokkan war ein Pionier der internationalen Sozialwissenschaft und einer der produktivsten Gestalten historisch-vergleichender Makrosoziologie. Der Artikel versucht, seine bleibende Bedeutung für die soziologische Europaforschung zu demonstrieren, obgleich die Europäische Union von heute kaum vergleichbar ist mit der Europäischen Gemeinschaft des Jahres 1979, dem Jahr seines frühen Todes. Die Europäische Union wird als neuer Versuch europäischer Zentrumsbildung verstanden. Im Anschluss an Rokkans typologisches Entwicklungsmodell wird die europäische Integration mit Prozessen, Strukturen und Konflikten in der Herausbildung von Massendemokratien und Wohlfahrtsstaaten kontrastiert. Insbesondere zwei Probleme der Europäischen Integration werden identifiziert: (a) die Unwucht zwischen Optionsrechten und Zugehörigkeiten und (b) das Fehlen interner Grenzziehung und Strukturierung.

Scblüsselwörter: Stein Rokkan; europäische Integration; Europäische Union; Konfliktstruktur; Makrosoziologie

Stefan Immerfall, Rights to root, rights to option and problems of supranational system building. Questions in the light of Sein Rokkan's macro-historical comparative sociology

Summary: Stein Rokkan, a leading promotor of international social sciences, was one of the most productive contributors to macro-historical comparative sociology. This article seeks to demonstrate his continuing relevance for analyzing European integration processes, even though the European Union of today is very different from the European Community of 1979, the year of Rokkan's untimely death. In The article perceives the European Union as a new stage in center building. Following Rokkan's developmental typology, European integration is contrasted with processes, structures and conflicts in European nation building. Two particular problems of the European Union are identified: (a) an imbalance of the »the right to root « and the »right to option «, and (b) deficiencies in boundary building and internal structuring of national conflicts.

Keywords: Stein Rokkan; European Integration; European Union; cleavage; macrosociology

* Meinem Lehrer Peter Steinbach zum siebzigsten Geburtstag. 
Stefan Immerfall, Dr. phil., ist Professor für Soziologie an der Pädagogischen Hochschule Schwäbisch Gmünd

Korrespondenzanschrift: Stefan.Immerfall@ph-gmuend.de

Um die Fruchtbarkeit sozialwissenschaftlicher Ansätze zu belegen, gibt es mehrere Wege. Im strikten Sinne wissenschaftlich einzig zulässig wäre es, sie gegen konkurrierende Ansätze auf Erklärungs- und Prognosekraft zu testen. Zweitens kann man aber auch versuchen, sie an bewährte Forschungstraditionen anzuschließen und ihre Kompatibilität mit den »Klassikern« eines Faches aufzuzeigen. Drittens kann man die in Frage stehende Theorie an neuartigen Forschungsproblemen und Fragestellungen erproben oder umgekehrt versuchen nachzuweisen, dass sie ein neues Licht auf alte Fragen wirft. Dieser letztgenannte Weg wird hier gewählt um Anschlussmöglichkeiten einer an Stein Rokkan orientierten, historisch-vergleichenden Makrosoziologie für die soziologische Europaforschung aufzuzeigen.

Stein Rokkan hat keine geschlossene Theorie, sondern eine Vielzahl, oft anlassbezogener Aufsätze hinterlassen. Dies mag damit zusammenhängen, dass er sich nicht nur für die Entwicklung überprüfbarer Modelle, sondern ebenso rastlos für den Aufbau einer materiellen Infrastruktur für die international-vergleichende Sozialwissenschaft eingesetzt hat. Dennoch verfügen wir mit der Kompilation Peter Floras und seiner einleitenden Rekonstruktion über einen bequemen Zugang zum Gesamtwerk Stein Rokkans ${ }^{1}$. Peter Flora hat denn auch frühzeitig auf Rokkans Analysepotential für den Integrationsprozess in Europa wie auch neue Grenzziehungen hingewiesen².

Dieser Gegenwartsbezug ist aber erneut zu vergegenwärtigen. Denn Stein Rokkan hat sich, wie noch zu zeigen sein wird, kaum über die zeitgenössische Europagemeinschaft geäußert. Er starb nach längerer Krankheit, mit 58 Jahren am 22. Juli 1979 in Bergen. Die Europäische Gemeinschaft von damals sah völlig anders aus als die Europäische Union von heute. Es handelte sich in der Hauptsache um eine Wirtschaftsgemeinschaft, aber die Entwicklung zu einer umfassenderen Union war erkennbar. Die innergemeinschaftlichen Zölle waren schon gefallen, die Beitrittsverträge mit Dänemark, Großbritannien, Irland und Norwegen unter Dach und Fach, der Ausbau zur Europäischen Union angepeilt und mit den verbliebenen EFTA-Mitliedern ein Freihandelsvertag geschlossen. Als einziges skandinavisches Land lehnte Norwegen im September 1972 den Beitritt zur EU in einer Volksabstimmung mit 53\% indes ab.

Trotz dieser Unterschiede, so die Behauptung dieses Aufsatzes, kann Rokkan helfen, Probleme der europäischen Integration besser zu verstehen. Zunächst wird kurz auf sein typologisches Entwicklungsmodell eingegangen. In ihrem Licht stellt sich die Eu-

1 Stein Rokkan, Staat, Nation und Demokratie in Europa. Die Theorie Stein Rokkans aus seinen gesammelten Werken rekonstruiert und eingeleitet von Peter Flora, Übersetzt von Elisabeth Fix, Frankfurt am Main 2000.

2 Peter Flora, »Externe Grenzbildung und interne Strukturierung - Europa und seine Nationen. Eine Rokkan'sche Forschungsperspektive« in: Berliner Journal für Soziologie 10, 2: S. 151165. 
ropäische Union als neue Etappe der Zentrumsbildung dar, die durch einen Mangel an interner Strukturierung nationsinterner und zwischennationaler Konflikte und Differenzen und durch ein Ungleichgewicht zwischen Optionsrechten und Zugehörigkeiten gekennzeichnet ist. Gerade an letzterem ist die EU nicht ganz unschuldig.

\section{Prozesse, Strukturen und Konflikte europäischer Herrschaftsbildung}

Stein Rokkans Modell der Herausbildung politischer Einheiten und der wachsenden Beteiligung breiter Bevölkerungsschichten umfasst historische Prozesse, Strukturen und Konflikte ${ }^{3}$. Zu den wichtigsten Prozessen gehört die Herausbildung von Staaten, Nationen, Massendemokratien und Wohlfahrtsstaaten:

1. Staatsbildung: Entstehung einer zentralen Herrschaft und einer administrativen Infrastruktur

2. Nationenbildung: Entstehen des Bewusstseins geteilter Gemeinsamkeiten

3. Massendemokratisierung: Einforderung und Gewährung politischer Repräsentation für immer breitere Schichten

4. Entstehung von Wohlfahrtsstaaten: Einführung und Erweiterung sozialer Rechte.

$\mathrm{Zu}$ den folgewirksamen Strukturen zählen historische Vorbedingungen wie z. B. Zeitpunkt und wirtschaftliche Basis der territorialen Konsolidierung, Grad der ethno-linguistischen und religiösen Homogenität und die Distanz zum europäischen Städtenetzwerk, die Rokkan in verschiedenen "geo-ökonomischen und geo-politischen Modellen« immer neu zu systematisieren versuchte ${ }^{4}$.

Rokkan wurde - nicht ganz zu Unrecht - vorgeworfen, dass er sich für die Handlungen der einzelnen Akteure, nicht interessiert hätte. Ein Strukturfunktionalist war er jedoch nicht, auch wenn er von den damals dominierenden Systemtheorien Talcott Parsons' stark beeinflusst war ${ }^{5}$. Dynamik erhalten Rokkans Konzepte durch die Konflikte, die die typisierten Akteursgruppen austragen. Konflikte begleiten, formen und bringen politische Einheiten hervor:

1. interne Konflikte und Koalitionen zwischen sozialen Gruppen und Spaltungsstrukturen, die langfristig über die jeweils präsenten Parteifamilien entscheiden

2. Konflikte um äußere Grenzziehung und interne Strukturierung bzw. Interaktion zwischen geographischen Räumen und Mitgliedschaftsräumen

Mit dem allgemeinen Modell ist keineswegs die Behauptung seiner Übertragbarkeit auf außereuropäische Entwicklungen verbunden. Möglichkeiten und Grenzen einer Übertragung müssen der historisch-empirischen Analyse vorbehalten sein.

3 Diese Konzepte beziehen sich auf die späteren Arbeiten Rokkans und vernachlässigen seine eher sozialpsychologischen Beiträge zur Wahl- und Einstellungsforschung.

4 Rokkan, Staat, Nation und Demokratie in Europa. Die Theorie Stein Rokkans aus seinen gesammelten Werken rekonstruiert und eingeleitet von Peter Flora. aaO. (FN 1), S. 170-178.

5 Stefan Immerfall, Territorium und Wablverhalten. Zur Modellierung geopolitischer und geoökonomischer Prozesse, Leverkusen 1992. 


\section{Die Europäische Union als neue Etappe der Zentrumsbildung}

Die Europäische Union stellt einen neuen Abschnitt in der Geschichte der territorialen Strukturierung Europas dar. Auch wenn bekanntermaßen die europäische Integration dem Vorbild der Nationalstaatsbildung nicht folgen kann - und erklärtermaßen nicht folgen will - lassen sich doch Parallelen ziehen. Meist werden in diesem Zusammenhang Vergleiche mit der US-amerikanischen Nationswerdung angestellt ${ }^{6}$. In gewisser Weise sind die historische Erfahrung der deutschen Nationaleinigung jedoch aufschlussreicher.

Während die jungen Staaten Amerikas trotz regionaler Besonderheiten und Konflikte eine gemeinsame Geschichte als Kolonien hatten, waren einige der 25 Gliedstaaten des 1871 gegründeten Deutsches Kaiserreichs in der Nationalstaatsbildung schon weit vorangeschritten. Zweifellos waren die sprachlichen und kulturellen Unterschiede zwischen seinen historischen Regionen kleiner als in der heutigen EU, aber weder sollten seine religiösen Konflikte unterschätzen werden noch die großen Unterschiede zwischen dem groß-agrarischen Osten und dem fortgeschrittenen Industriekapitalismus im Westen und in der Mitte, die sich als große Belastung erweisen sollte. Dank des allgemeinen und geheimen Wahlrechts für Männer ab 25 Jahren erlauben wahlstatistische Analysen der Reichstagswahlen einige Aussagen darüber, wie die regionalen Öffentlichkeiten dennoch allmählich auf das Zentrum orientiert wurden ${ }^{7}$.

Die wichtigsten politischen Strömungen hatten sich lange vor der Reichsgründung auf landesstaatlicher Ebene herausgebildet. Obwohl die regionalspezifischen Prägungen weiterhin bestanden, mussten die Abgeordneten und Parteien in den Konflikten um die Reichsgesetzgebung rasch ihre Haltung zum neuen Kaiserreich bestimmen, auch wenn die Reichsgründung keineswegs überall auf Zustimmung stieß. Mit den neuen reichspolitischen Herausforderungen in der Steuer-, Sozial- und Wirtschaftspolitik, sowie den konfessions- und kulturpolitischen Auseinandersetzungen in Kirchenkampf und Schulstreit bildete sich so etwas wie ein nationalstaatliches Parteiensystem heraus $^{8}$. Nicht in jeder politischen Strömung führte die wachsende Nationalisierung der Wahlkonflikte gleichermaßen zu einer Entregionalisierung des Wählerverhal-

6 Anand Menon / Martin A. Schain (Hg.), Comparative Federalism. The European Union and the United States in Comparative Perspective, Oxford 2006.

Jens Alber / Neil Gilbert (Hg.), United in Diversity? Comparing Social Models in Europe and America., Oxford 2010.

7 Peter Steinbach, »Nationalisierung, soziale Differenzen und Urbanisierung als Bedingungsfaktoren des Wahlverhaltens im Kaiserreich« in: Historical Social Research, 1990, 15(2), S. 63 82.

8 Peter Steinbach, »Wahlrecht und Verfassungsentwicklung in Deutschland von der Revolution 1848/49 bis zum Ende des Kaiserreichs « in: Detlef Lehnert (Hrsg.) Wabl- und Stimmrechtskonflikte in Europa. Ursprünge - Neugestaltungen - Problemfelder, Berlin 2018, S. 111-147. Immerfall / Steinbach "Politisierung und Nationalisierung deutscher Regionen im Kaiserreich « in: Berg-Schlosser D., Schissler J. (Hg.) Politische Kultur in Deutschland. In: Politische Vierteljabresschrift, Sonderheft 18, S. 68-79. 
tens. ${ }^{9}$.Es war ausgerechnet die als »Reichfeind « verfemte Sozialdemokratie, die - funktional gesehen - zur ersten »nationalen Partei« wurde. Indem sie das relative Gewicht der Klassen- gegenüber territorial-kulturellen Spaltungen vergrößerte, trug sie - ungewollt - zur Nationalisierung der Bevölkerung bei.

Zweifellos sind die Mitgliedstaaten der Europäischen Union ungleich »widerborstiger« als es die Bundesstaaten des Deutschen Kaiserreichs waren, vom erdrückenden Übergewicht Preußens ganz abgesehen. Auch wurden die »Schwellen der Demokratisierung «10 längst überschritten, so dass die Strategie der Europäisierung durch »Fundamentalpolitisierung « entfällt. Die EU gebietet zudem nicht über die beiden altbewährten Instrumente, ältere Identitäten und Loyalitäten abzulösen: Armee und Schule. Dennoch ließe sich argumentieren, mit dem direkt gewählten Europäischen Parlament verfüge die Europäische Union mittlerweile auch über einen »plebiszitäre Wahlkanal«, der die nationalen politischen Massenmärkte fokussieren könnte.

Wenn sich aber trotz ähnlicher Voraussetzungen wie seinerzeit in den Monarchien des 19. und 20. Jahrhundert keine Parlamentarisierung einstellen will, könnte es an den fehlenden gesellschaftlich-politischen Voraussetzungen liegen ${ }^{11}$. Eine klassische Opposition gibt es nicht und den in den Fraktionen zusammengeschlossen Europäischen Parteien fehlt die gesellschaftliche Verwurzelung. Die Wähler wissen nicht, wie sich ihre Stimmen auf die EU-Politik auswirkt, da sie es mit Gewaltenkonglomerat zu tun haben, dessen Verantwortlichkeiten unklar sind. Obwohl in der aktuellen Europawahl die Europäische Volkspartei und die Sozialdemokratie eine deutliche Niederlage erlitten haben, wird die große Koalition EU-freundlicher Parteien fortbestehen. Den konsenssuchenden Fraktionen der Mitte mangelt es an Kraft zu Politisierung: Kritiker der Machtausweitung der EU werden als »Ungläubige exkommuniziert « ${ }^{12}$.

Wenngleich Rokkan dem nationalstaatlichen Wohlfahrtsstaat als erfolgreiches Modell territorialer Einheit besondere Beachtung geschenkt hat, stellt er eine Etappe, keinen Abschluss der europäischen Konfliktgeschichte dar. Maurizio Ferrera ${ }^{13}$ hat Rokkans Skepsis gegenüber der Übertragung nationaler Loyalitäten auf eine supranationale Einheit herausgearbeitet ${ }^{14}$. Diese Skepsis war sicher auch von Norwegens stolzen und unerwarteten »Nein« zum Beitritt beeinflusst, das sich 1994 wiederholte

9 Stefan Immerfall, »Wahlverhalten und Parteiensystem im Kaiserreich: einige quantitative Befunde « in: H. Best (Hrsg.), Politik und Milieu. Wabl- und Elitenforschung im historischen und inter-kulturellen Vergleich, Sankt Katharinen 1989, S. 34 - 59.

10 Rokkan, Staat, Nation und Demokratie in Europa. Die Theorie Stein Rokkans aus seinen gesammelten Werken rekonstruiert und eingeleitet von Peter Flora, aaO. (FN 1), S. 296.

11 Peter Graf Kielmansegg, Wohin des Wegs, Europa? Beiträge zu einer überfälligen Debatte, S. 153, Baden-Baden 2015.

12 Luuk van Middelaar, Alarums and Excursions. Improvising politics on the european stage. De Newcastle, 2019, S. 245; ähnl. Dermot Hodson and Uwe Puetter, »The European Union in disequilibrium. New intergovernmentalism, postfunctionalism and integration theory in the post-Maastricht period. «Journal of European Public, published online: 17 Jan 2019, S. 24.

13 Maurizio Ferrera, »Disproved or vindicated? Stein Rokkan's 'impossibility theorem' on welfare democracy and European integration. « in: Journal of European Social Policy 29 (1), S. 3-12.

14 Ferrera (2018) spricht allerdings von einem »Unmöglichkeits-Theorem «, wo Rokkan (Votes count, resources decide. Refleksjoner over territorialitet versus funksjonalitet I norsk og eu- 
(Schweden blieb somit das letzte wohlhabende Land, das der EU beigetreten ist). In der Hauptsache dürfte sie aber ein Ergebnis seines Studiums der Prozesse der Demokratisierung sein. Rokkans Werk enthält systematische Informationen darüber, wie neue Solidaritäten und einheitliche politische Systeme entstehen. Somit können wir ihnen umgekehrt Hinweise auf die Schwierigkeiten entnehmen, mit welchen die Übertragung substantieller Autoritär auf eine supranationale Gemeinschaft zu rechnen hat.

Ausgangspunkt der Überlegungen ist der nationalstaatliche Wohlfahrtsstaat als Ergebnis einer jahrhundertelangen Konfliktgeschichte. Zwar lassen sich ökonomische Vereinigungen grenzüberschreitend aufbauen und supraterritoriale Allianzen hatten ihre Vorgänger schon in der Hanse und später dann im atlantischen Kapitalismus. Doch nur die Nation wurde zur bestimmenden Gemeinschaft. Der moderne Staat ist nicht nur ein politischer Akteur unter vielen. Er unterscheidet sich von seinen »kooperativen Partnern « (Unternehmen, Verbände, Interessenorganisationen) nicht nur durch seinen Anspruch auf ein Territorium, sondern - als Wohlfahrtsstaat - auch durch seinen Anspruch, die Solidargemeinschaft seiner Bürger zu repräsentieren.

Dieser Anspruch mag ideologisch überhöht sein. Er findet seinen konkreten Ausdruck jedoch in der Wahlarena, in der die abgegebenen Stimmen mit gleichem Gewicht zum Ausdruck gebracht werden und sich in den sozialen Rechten und Sicherungen niederschlagen. Während die kooperativen Gruppen (insbesondere die Unternehmen) mit Abwanderung und Ressourcenverlagerung drohen können, verbleibt den Volksschichten nur dieser »plebiszitäre Wahlkanal«. Sie sind daher ungleich stärker auf den Territorialstaat angewiesen.

\section{Grenzziebung und interne Strukturierung}

Die extreme Diversität innerhalb Europas hat keineswegs die Herausbildung eines gewissen wechselseitigen Vertrauens der Europäischen Völker und Bürger verhindert, im deutlichen Kontrast zur Zwischenkriegszeit ${ }^{15}$. Verschiedene Umfragen ${ }^{16}$ legen sogar eine

ropeisk politick, pp. 216-224 in: Dahl, Ottar m. fl. (red.) Makt og motiv. Et festskrift til Jens Arup Seip 1905-1975. Oslo 1975., S. 223) nur vor der "Unwahrscheinlichkeit « spricht [Hinweis: Maurizio Ferrara [2018] bezieht sich auf die italienische Übersetzung: Rokkan, Stein, 1975. I voti contano, le risorse decidono. Rivista Italiana di Scienza Politica, 5, 1: 167-176; der Beitrag ist in Teilen in Floras Rekonstruktion aufgenommen worden: Rokkan aaO. (FN 1), S, 316-322].

15 Jan Delhey / Gregori Dragolov, »Happier together. Social cohesion and subjective wellbeing in Europe. «In: International Journal of Psychology 51 (2016), S. 163-176.

Wolfgang Glatzer / Jürgen Kohl »The History of Well-Being in Europe« in: Estes R., Sirgy M. (eds) The Pursuit of Human Well-Being. International Handbooks of Quality-of-Life., S. 409-452, Cham 2018.

16 Irina Ciornei / Ettore Recchi, »At the Source of European Solidarity. Assessing the Effects of Cross-border Practices and Political Attitudes. «In: Journal of Common Market Studies 2017, 55(3), S. 417-671.

Jürgen Gerhards / Holger Lengfeld / Zsófia S. Ignácz / Florian Kley / Maximilian Priem. 2018. "How Strong is European Solidarity? «In: Berliner Studien zur Soziologie Europas (BSSE) 37: 2018, FU Berlin, Institut für Soziologie. 
hohe Bereitschaft der Europäer zur transnationalen Umverteilung nahe. Selbst, wenn dieser Befund zum Nennwert akzeptiert wird ${ }^{17}$, bleibt die Tatsache, dass trotz der Schaffung einer gemeinsamen Wirtschafts- und Währungsunion weder eine Konvergenz zu einem einheitlichen Modell eines Europäischen Wohlfahrtsstaates erkennbar ist noch eine Ergänzung oder gar Ablösung der nationalen durch ein gesamteuropäisches Wohlfahrtsystem. Die soziale Dimension der EU ist mehr Rhetorik als Realität ${ }^{18}$. Von allen Elementen, die Rokkan ${ }^{19}$ für die vierte Phase in der Ausweitung des territorial-staatlichen Verwaltungsapparates benannt hat: Zunahme von Umverteilungsinstanzen, Aufbau öffentliche Wohlfahrtsdienste, Entwicklung von nationenweiten Politiken zur Angleichung der Soziallagen (progressive Besteuerung und Umverteilung) und Transfers von den reicheren zu den zurückgebliebenen Regionen - ist auf europäische Ebene mit den Sozial- und Strukturfonds nur letzteres ansatzweise implementiert.

In den Nationalstaaten hing die Ausweitung der Umverteilung engt mit den funktionalen Folgen der industriellen Revolution zusammen, das heißt dem Stadt-Land und dem Klassen-Konflikt. Der Aufbau des Wohlfahrtstaates wird als »interne Strukturierung « verstanden, als Kanalisierung der Klassenkonflikte durch Einbeziehung der organisatorischen und politischen Vertretungen der ärmeren Bevölkerungsschichten und der zurückgebliebenen Regionen in das politische System. Treibende Kräfte waren auf korporative Ebenen vor allem Gewerkschaften und Bauernorganisationen, im plebiszitären Kanal Arbeiterparteien und christlich-konservativ Parteien. Damit verbunden war die allmähliche Ausbildung nationaler Identitäten und die staatsbürgerliche Ausweitung des Solidargedankens Der wohlfahrtstaatliche Nationalstaat stellt dem Anspruch nach die räumliche Basis für ein Zusammengehörigkeitsgefühl da. Er sichert wechselseitige Verpflichtungen institutionell und eröffnet damit die Chance, dass sich der Kreis des Vertrauens über die Eigengruppe hinaus weitet.

Diese interne Strukturierungsleistung ist auf europäischer Ebene kaum erkennbar ${ }^{20}$. Über die schwache Verankerung der Europäischen Parteien wurde schon gesprochen. Auch die nationalen Gewerkschaften tun sich mit gemeinsamen Aktionen schwer. Am ehesten gelingt es den Bauern: kein Wunder, wenn man bedenkt, dass die Agrarpolitik von Anbeginn der am stärksten vergemeinschaftete Bereich war und bis heute 40 Prozent des EU-Haushaltes in den Agrarbereich fließen. EU-Bürgerschaftsrechte - etwa der Zugang von Arbeitsmigranten zu den nationalstaatlichen Sicherungssystemen wurden vielfach nicht erstritten, sondern durch Richterrecht, durch den EuGH, geschaffen und ausgeweitet. Sie haben allerdings keine Umverteilung zwischen Klassen

17 Kritisch: Stefan Immerfall, »Mehr Solidarität durch >Mehr Europa«? « in: Wolfgang Aschauer / Elisabeth Donat / Julia Hofmann (Hg.), Solidaritätsbrüche in Europa: Konzeptuelle Überlegungen und empirische Befunde, Wiesbaden 2016, S. 49-71.

18 Martin Seeliger, »Warum die EU aus gewerkschaftlicher Sicht keine Solidargemeinschaft darstellt.« In: Leviathan, 2017, 45(4), S. 438-458.

19 Rokkan 2000. Staat, Nation und Demokratie in Europa. Die Theorie Stein Rokkans aus seinen gesammelten Werken rekonstruiert und eingeleitet von Peter Flora, aaO. (FN1), S. 168.

20 Maurizio Bach, »Unbounded Cleavages, Grenzenabbau und die Europäisierung sozialer Ungleichheit« in: Monika Eigmüller / Georg Vobruba (Hg.), Grenzsoziologie. Die politische Strukturierung des Raumes, Wiesbaden 2006, S. 145-156. 
zum Gegenstand, sondern eine Umverteilung innerhalb der mittleren und unteren Schichten verschiedener Mitgliedsstaaten: »Die niedrigschwellige Gleichbehandlung von EU-Bürgern hat eine spiegelbildliche Diskriminierung der Inländer zur Folge, wenn sie disproportional zu Finanzierung der Leistungen heranzogen werden. ${ }^{21}$. Gleiche Behandlung in einem sozial hochgradig ungleichen Raum des Rechtes kann leicht Befürchtungen des Sozialtourismus provozieren und die Legitimation des europäischen politischen Systems insgesamt in Frage stellen ${ }^{22}$.

Nach Rokkan besitzen die moralischen Verpflichtungen zur wechselseitigen Hilfe, die sich im Nationalstaat herausgebildet haben, eine territoriale Basis. Sie beruhen auf der »Interaktion zwischen internen und externen Grenzbildungsstrategien ${ }^{23}$. Im gleichen Zusammenhang spricht Paul Collier ${ }^{24}$ von einem Koordinierungsproblem: es sei ein großer Schritt von der schwachen Kraft des Altruismus und den Pflichten zur Hilfeleistung für Nahestehende zur allgemeinen Reziprozität, die z. B. Menschen dazu bringt, hohe Steuersätze zu akzeptieren. Er bedarf eines Narratives, das uns Zugehörigkeit und Normen des Gemeinsinns vermittelt. Wie Rokkan nimmt Collier ${ }^{25}$ an, dass dieses wirkmächtige Narrativ hauptsächlich auf die Nation bezogen ist. Auch wenn dieses Narrativ konstruiert ist, so ist es doch nicht beliebig und vor allem nicht beliebig ersetzbar.

\section{Kippt die Balance zwischen Optionsrechten und Zugehörigkeiten?}

Die europäische Integration hat den sozialrechtlich geschlossenen Nationalstaat perforiert. Sie kann als vor allem ökonomische Grenzüberschreitung konzipiert werden ${ }^{26}$. Weniger erfolgreich waren die Strategien der Grenzbildung. Die politische Mitgliedschaft wurde erweitert, womit neue Rechte einhergingen. Die soziale Mitgliedschaft blieb aber im wesentlichen weiterhin nationalstaatsbezogen; die Europäische Gemeinschaft konnte die sozialstaatlichen Institutionen des Nationalstaates nicht ersetzen. Zwar kam es entgegen Rokkans Erwartung zu einer substantiellen Übertragung verschiedener Hoheitsrechte auf die supranationalen Institutionen der EU. Die Übertragung hat jedoch genau die Konflikte provoziert, die Rokkan erwartet hat. ${ }^{27}$

21 Susanne K. Schmidt, »Der Brexit: ein Dilemma auch für die Europäische Union« in: Frankfurter Allgemeine Zeitung vom 18.2.2019, S. 6.

22 Susanne K. Schmidt / Michael Blauberger / Dorte Martinsen, » Free movement and equal treatment in an unequal union « in: Journal of European Public Policy 25, 10, 2018: S. 1391-1402.

23 Rokkan, Staat, Nation und Demokratie in Europa. Die Theorie Stein Rokkans aus seinen gesammelten Werken rekonstruiert und eingeleitet von Peter Flora, aaO. (FN1), S. 281.

24 Paul Collier, Sozialer Kapitalismus! Mein Manifest gegen den Zerfall unserer Gesellschaft. München 2019, S. 83.

25 Collier, Sozialer Kapitalismus! Mein Manifest gegen den Zerfall unserer Gesellschaft. aaO. (FN 24), S. 93.

26 Rokkan, Staat, Nation und Demokratie in Europa. Die Theorie Stein Rokkans aus seinen gesammelten Werken rekonstruiert und eingeleitet von Peter Flora, aaO. (FN1), S. 134.

27 Ferrera, »Disproved or vindicated? Stein Rokkan's 'impossibility theorem' on welfare democracy and European integration « in: Journal of European Social Policy 29 (1), S. 3-12. 
Sie wurde durch Globalisierungsprozesse verstärkt und hat zur gegenwärtigen Krise der Repräsentativität beigetragen. Ob diese Krise ihre Wurzeln stärker in dem Empfinden mangelnder Anerkennung ${ }^{28}$, in handfesten ökonomischen Gründen ${ }^{29}$ oder in einer emotionalen Reaktion auf einen ökonomischen und sozialen Epochenbruch ${ }^{30}$ hat, sei dahingestellt. Paul Collier hat die Interaktion von kultureller und ökonomischer Verunsicherung in einem kleinen Modell sehr schön deutlich gemacht ${ }^{31}$. Dieses Modell zeigt vereinfachend, wie wenig es bedarf, einen Zustand, in dem auch die Besserverdienenden ihr Ansehen überwiegend aus ihrer nationalen Identität beziehen, in einem $\mathrm{Zu}$ stand steigender Polarisierung überzuführend, der das Prinzip der Wechselseitigkeit untergräbt.

Dies ist dann der Fall, wenn die besonders erfolgreichen und höher Qualifizierten ihre Selbst-Wertschätzung zunehmend aus ihrem Beruf und ihrer Qualifikation beziehen. Den Zurückgebliebenen bleibt nichts anderes übrig, als sich weithin an die Nation zu klammern, die aber zunehmend an Wertschätzung verliert. Eine Abwärtsspirale kommt in Gang: die geringer qualifizierten Schichten - sowohl in den Metropolen als auch in die abgehängten ländlichen Regionen - entwickeln Ressentiments, während die höher Gebildeten für sich in Anspruch nehmen, jenen moralisch überlegen zu sein ${ }^{32}$. In dem Maße, wie sich die an Qualifikation einerseits und an Nationalität andererseits orientierten Identitäten voneinander entfernen, beginnt das Vertrauen in die Menschen an der Spitze der Gesellschaft zusammenzubrechen und das Gefühl der Zugehörigkeit und der Verpflichtung zu erodieren. Es ist jener gebildete, qualifizierte Teil der Bevölkerung, welcher die Nationalität immer weniger als Kern seiner Identität betrachtet, der die Europäische Integration besonders befürwortet ${ }^{33}$. Die Europäische Union läuft somit Gefahr, als reines Elitenprojekt identifiziert werden. ${ }^{34}$

28 Francis Fukuyama, Identity. The demand for dignity and the politics of resentment. New York 2018.

29 Philip Manow, Die politische Ökonomie des Populismus, Bd. 2728. Erste Auflage, Sonderdruck, Originalausgabe, Berlin 2018.

30 Cornelia Koppetsch, Gesellschaft des Zorns. Rechtspopulismus im globalen Zeitalter. Bielefeld 2019.

31 Collier, Sozialer Kapitalismus! Mein Manifest gegen den Zerfall unserer Gesellschaft, aaO. (FN 24), S. 78-82.

32 Klaus Kraemer, "Sehnsucht nach dem nationalen Container. Zur symbolischen Ökonomie des neuen Nationalismus in Europa.«In: Leviathan 46, 2, 2018, S. 280-302.

Carlo Strenger, Diese verdammten liberalen Eliten. Wer sie sind und warum wir sie brauchen. Berlin 2019.

33 Stefan Immerfall / Klaus Boehnke / Dirk Baier, "Identity ", in: Handbook of European Societies. Social Transformations in the 21st Century, Stefan Immerfall / Göran Therborn (Hg.), New York 2010, S. 325-353. Theresa Kuhn / Erika van Elsas / Armen Hakhverdian / Wouter van der Brug, » An ever wider gap in an ever closer union: Rising inequalities and euroscepticism in 12 West European democracies, 1975-2009«. In: Socio-Economic Review 14, 2014, S. 27-45. Alina Polyakova / Neil Fligstein. »Is European integration causing Europe to become more nationalist? Evidence from the 2007-9 financial crisis. «In: Journal of European Public Policy 23, 2016, S. 60-83.

34 Max Haller, Die Europäische Integration als Elitenprozess. Das Ende eines Traums?, Wiesbaden 2009. 
Begleitet und verstärkt wird diese Spaltung vom Niedergang der Sozialdemokratie, die immer mehr die Rechte von allen möglichen, gesellschaftlich benachteiligten Gruppen einfordert, sich von der "praktischen Reziprozität « ${ }^{35}$ indes häufig entfernt. ${ }^{36}$ Angesichts der von Rokkan früh identifizierten Zangenbewegung von Globalisierung und Ethnisierung ${ }^{37}$, brauchen wir beides: das »Recht auf Wurzeln « und das »Recht auf Wahlmöglichkeiten «38. Rokkan löste mit diesem Begriffspaar»das Dilemma eines Sozialwissenschaftlers, der täglich die Nachwirkungen von Traditionen verspürte und zugleich wusste, dass nur deren Infragestellung und Veränderung Entwicklungen ermöglichte ${ }^{39}$. Der europäische Integrationsprozess hat erfolgreich Grenzen geöffnet und Barrieren beseitigt. Mit Rokkan müssen wir heute die Frage stellen, ob die Europäische Union die Balance zwischen Optionsrechten und Zugehörigkeiten wahren kann.

35 Collier, Sozialer Kapitalismus! Mein Manifest gegen den Zerfall unserer Gesellschaft, aaO. (FN 24), S. 23.

36 Jane Gingrich / Silja Häusermann, »The decline of the working-class vote, the reconfiguration of the welfare support coalition and consequences for the welfare state in: Journal of European Social Policy 25 (1), 2015, S. 50-75.

37 Stefan Immerfall / Peter Steinbach, »Historisch-vergleichende Makrosoziologie: Stein Rokkan - der Beitrag eines Kosmopoliten aus der Peripherie in: Stefan Immerfall and Peter Steinbach (Hg.), Historisch-vergleichende Makrosoziologie: Stein Rokkan - der Beitrag eines Kosmopoliten aus der Peripherie. Historical Social Research Vol. 20 (1995) S. 4-7 No. 2: Special Issue:.

38 Rokkan 2000. Staat, Nation und Demokratie in Europa. Die Theorie Stein Rokkans aus seinen gesammelten Werken rekonstruiert und eingeleitet von Peter Flora, aaO. (FN1), S. 212. Stein Rokkan / Derek W. Urwin, Politics of Territorial Identity: Studies in European Regionalism. London 1982, S. 15.

39 Stefan Immerfall / Peter Steinbach, »Historisch-vergleichende Makrosoziologie: Stein Rokkan - der Beitrag eines Kosmopoliten aus der Peripherie«aaO. (FN 37), S. 6. 